\title{
不同人为干扰条件下毒药树种群数量特征的比较
}

\author{
陈国科 12 彭 $\quad$ 华 ${ }^{*}$ \\ （1 中国科学院昆明植物研究所, 昆明 650204）（2 中国科学院研究生院，北京 100039）
}

摘 要 云南是毒药树 (Sladenia celastrifolia) 的分布中心, 日益严重的人为干扰不可避免地影响毒药树种群的数量 特征。基于毒药树年龄与胸径之间的关系, 初步分析了受人为干扰程度不同的两个种群的年龄结构、静态生命表 和存活曲线, 并对这两个种群进行了比较。严重受干扰的种群的幼苗库相对不足, I 龄级个体数比例为 $12.7 \%$, II 、III、IV龄级个体数所占比例为 $80.3 \%$ 种群衰退趋势明显; 未受干扰的种群幼苗库相对充足, 年龄结构呈现稳定 种群的特征。这两个种群的生命表也存在差异, 其中最明显的就是严重受干扰的种群的 I 龄级和 II 龄级的死亡率 为负, 这是幼苗库不足的一个直接反映。两个种群的存活曲线都表现出低龄级个体死亡率高的特征, 但未受干扰 的种群各龄级个体的死亡率的变化幅度较小。毒药树种群的各项特征与鹅掌楸 (Liriodendron chinense) 及其它几种 濒危植物的相关特征相似。虽然毒药树还未被列为受威胁的物种, 但它已具备 IUCN 所定义的受威胁物种的特点, 因此，对它的保护已十分必要, 除了在野外建立相应的毒药树保护点之外, 在植物园中培植人工种群也是必须的。

关键词 人为干扰 种群数量特征 年龄结构 生命表 存活曲线

\section{QUANTITATIVE CHARACTERISTICS OF TWO POPULATIONS OF SLADENIA CELASTRIFOLIA UNDER DIFFERENT LEVELS OF HUMAN DISTURBANCE}

\author{
CHEN Guo-Ke ${ }^{12}$ and PENG Hua ${ }^{1 *}$ \\ (1 Kunming Institute of Botany, Chinese Academy of Sciences , Kunming 650204 , China) \\ (2 Graduate School of Chinese Academy of Sciences , Beijing 100039 , China)
}

\begin{abstract}
Sladenia celastrifolia has a scattered distribution in Southwest China, especially in Yunnan Province. However, characteristics of many populations have been largely altered by increasing human activities. Using age-diameter relationships, we analyzed the age structure, the life table and the survival curves of two populations of this species, which have been affected by human disturbance to different degrees. In the heavily disturbed population, there were fewer young-aged individuals. The individuals of the age class I account for only $12.7 \%$, whereas those of the age class II , III , and IV together account for $80.3 \%$ of the total individuals of the population , indicating a declining population. In contrast, the age structure of the less disturbed population appeared to be stable with sufficient numbers of young-aged individuals. The life tables of the two populations were different : the death rates of the age class I and II were negative, indicating a shortage of young-aged individuals. The survival curves of both populations were characterized by high death rates of young-aged individuals, while the death rates of all age classes of the less disturbed population were stable. The age structure and dynamics of the two populations of this species are similar to those of some other endangered species in China, such as Liriodendron chinense and Cathaya argyrophylla. Although S. celastrifolia has not been regarded as an endangered species until now, it has attributes typical of an endangered species according to the standards of IUCN. Therefore, more attention should be paid for the conservation of this species such as establishing nature reserves in its original habitat as well as ex-situ conservation efforts.
\end{abstract}

Key words Human disturbance, Population quantitative characteristics , Age structure , Life table , Survival curve

毒药树 (Sladenia celastrifolia) 分布于云南大部分 地区、贵州西南部、广西西部 境外泰国、缅甸也有，
主要分布在中国云南，常见于热带 (760 1 $100 \mathrm{~m}$ )、 亚热带海拔 $1100 \sim 1900 \mathrm{~m}$ 的沟谷常绿阔叶林中 
(李璐, 2001)。由于树形优美、常绿, 开花时香味浓 郁, 而且花期 3 个月, 毒药树已经被云南省的多个城 市确定为重要的绿化树种。

云南是中国生物多样性的关键地区之一(蒋志 刚等, 1997)，人类的经济发展已对当地的生态系统 造成了很大的破坏 (郭辉军,1998)。人类活动对地 球环境的干扰是多方面的, 在个体、种群、群落及生 态系统等各个等级都有明显的反映 (Scariot,1999; Wang et al.,2001; Bruna，2003)。我们对毒药树种 群的实际调查结果表明: 人为干扰已经对该物种造 成了严重的破坏, 其生存受到了很大的威胁。为了 对该物种的生存状况有一个合理的评价, 本文从种 群数量特征的角度出发, 对受人为干扰程度不同的 两个毒药树种群的年龄结构、生命表以及存活曲线 进行了比较研究, 目的是阐明人为干扰对毒药树种 群数量特征的影响, 为毒药树的保护和可持续利用 提供理论依据。

\section{1 研究方法}

\section{1 研究样地}

查阅昆明植物所标本馆 (KUN) 毒药树的标本记 录，对记录上的每个分布点进行实际调查。调查结 果表明, 毒药树种群的分布点在逐级减少, 而且种群 规模也很小, 所调查的种群中含个体数在 100 株以 上的种群仅有 2 个, 含个体数在 50 株以上 100 株以 下的种群有 3 个。本文进行对比研究的两个种群均 处于常绿阔叶林中有水的沟谷地带 (表 1 ) ,但受人 为干扰的程度明显不同。其中一个种群位于易门县 森林公园外的水库边 (为了叙述方便，我们简称其为 种群 A) ;另一个种群位于禄劝普渡河村附件的峡谷 地带 (简称为种群 $\mathrm{B}$ )。

从 1986 年到 2000 年,易门森林公园在建设过 程中, 由于景区道路和大量观赏景点的修建, 种群 A
的生境遭到了很大的破坏。群落的乔木层以滇青冈 (Cyclobalanopsis glaucoides) 毒药树占优势，总盖度达 $60 \%$ 灌木层平均高度为 $1.5 \mathrm{~m}$, 层盖度约为 $20 \%$ ， 主要有以下几种 :滇青冈的幼苗、云南木樨榄 (Olea yunnanensis)、小叶青冈(Cyclobalanopsis myrsinaefolia) 和滇南杜鹃 (Rhododendron hancockii) 等。

种群 B 所在地山势陡峭, 生境受人为干扰的痕 迹极少，尽管普渡河流域具有干热河谷的气候特点， 但种群 B 所处峡谷较为湿润, 群落结构也相对比较 完整。乔木层以毒药树和滇青冈占优势, 盖度达 $80 \%$ 以上 林下由于乔木层的郁闭而极少见灌木 林 缘处的灌木层比较发达,主要有以下几种:白枪杆 (Fraxinus malacophylla)、黑背鼠李 (Rhamnus nigricans)、滇鼠刺 ( Itea yunnanensis) 和一把香 (Wikstroemia dolichantha) 等。

凭证标本均存放于昆明植物研究所标本馆 $(\mathrm{KUN})$ 。

\section{2 树龄的确定}

参照吴承祯和洪伟 (2002) 的方法, 我们在 A、B 两种群以 10 为胸径梯度, 每一胸径梯度内用树木生 长雉取两棵树的树芯, 每棵树在离地面高 $1.3 \mathrm{~m}$ 的 地方从互相垂直的两个方向取树芯, 每一胸径梯度 内共钻取 4 个树芯，带回试验室分析，确定树木的具 体年龄。以测定的年龄和胸径的数据为依据, 分别 建立两个种群的胸径与年龄之间的回归方程 :

$$
\begin{aligned}
& Y=0.0001 D^{3}-0.0014 D^{2}+0.4469 D \\
& +6.5021\left(r^{2}=0.983, p<0.05\right) \\
& Y=0.0001 D^{3}-0.0086 D^{2}+0.9951 D \\
& -0.2663\left(r^{2}=0.993, p<0.05\right)
\end{aligned}
$$

$Y$ 为年龄, $D$ 为胸径,式 (1) (种群 A) 和式 (2) (种群 B)的拟合效果均显著(图 1)。种群中所有个 体的年龄均按上述两式来确定。

表 1 两个种群的生境概况

Table 1 Habitat conditions of two populations of Sladenia celastrifolia

\begin{tabular}{ccccc}
\hline $\begin{array}{c}\text { 种群 } \\
\text { Population }\end{array}$ & $\begin{array}{c}\text { 海拔 } \\
\text { Elevation }(\mathrm{m})\end{array}$ & $\begin{array}{c}\text { 坡度 } \\
\text { Slope }\left(^{\circ}\right.\end{array}$ & $\begin{array}{c}\text { 坡向 } \\
\text { Aspect }\end{array}$ & $\begin{array}{c}\text { 生境 } \\
\text { Habitat conditions }\end{array}$ \\
\hline $\mathrm{A}$ & 1910 & 30 & 西南 SW & $\begin{array}{l}\text { 水库边 常绿阔叶林 } \\
\text { Lakeshore , evergreen broadleaved forest } \\
\text { 水沟边 常绿阔叶林 } \\
\text { Stream sides , evergreen broadleaved forest }\end{array}$ \\
\hline B & 1820 & 45 & 东南 SE & .
\end{tabular}



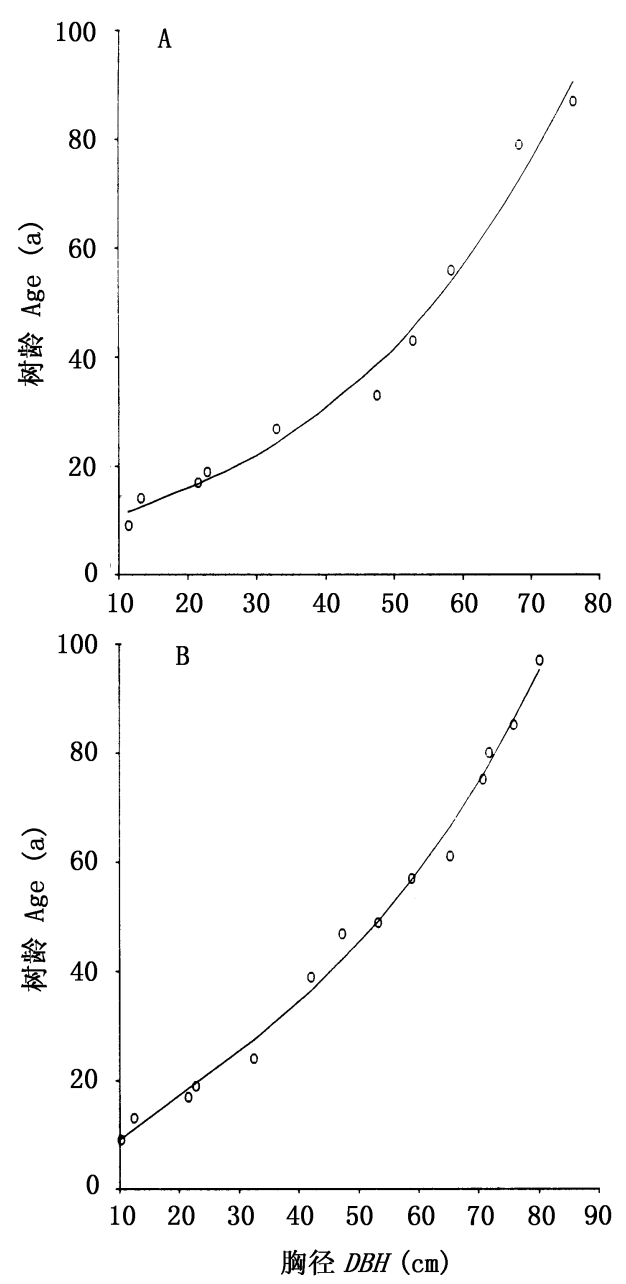

图 1 毒药树种群年龄与胸径的关系

Fig. 1 Relationship between age and DBH of Sladenia celastrifolia

A : 重度干扰种群 Heavily disturbed population B : 无干扰种群 Unaffected population

\section{3 年龄结构图的绘制}

采用等距年龄间隔，每 10 年为一个龄级，每一 龄级所代表的具体年龄阶段如下: I 龄级 $(0 \sim 10$ 年)、II 龄级 (10 20 年)、III龄级 (20 30 年)、IV 龄 级 (30 40 年)、V龄级 $(40 \sim 50$ 年)、VI龄级 $(50 \sim 60$ 年)、VII龄级 $(60 \sim 70$ 年)、VIII龄级 (70 80 年) 和 IX 龄 级 $(80 \sim 90$ 年)。以龄级为横坐标, 以各龄级的个体 数为纵坐标绘制年龄结构图 (图 2)。

\section{4 特定时间种群生命表和存活曲线}

参照江洪 (1992)的方法绘制两个种群的生命表 和存活曲线。表中具体包括以下各项内容 : $x$ 为龄 级中值; $a_{x}$ 为 $x$ 龄级开始时的实际存活数; $l_{x}$ 为 $x$ 龄级开始时的标准化存活数; $d_{x}$ 为从 $x$ 到 $x+1$ 期间 的标准化死亡数; $q_{x}=d_{x} / l_{x} ; T_{x}$ 为龄级 $x$ 及其以上 各龄级的个体存活总数; $e_{x}$ 为进入 $x$ 龄级个体的平
均生命期望, $e_{x}=T_{x} / l_{x}$ 。 以生命表中的标准化存活 数 $l_{x}$ 为纵坐标, 以龄级为横坐标, 绘制种群的存活 曲线(图 3)。

\section{2 实验结果}

\section{1 毒药树种群年龄结构}

图 2 是两个毒药树种群的年龄结构。从图中我 们可以看出, 种群 $\mathrm{A}$ 的各龄级个体分布很不均匀, I龄级个体相对很少，占各龄级总数的 $12.7 \%$,该 种群的 II 龄级、 III 龄级和 IV 龄级的个体占绝对优势， 占各龄级总数的 $80.3 \%$, 种群发展所需的幼苗库相 对不足。而种群 $\mathrm{B}$ 各龄级的个体比例波动不大, I 龄级个体相对比较充足，占各龄级总数的 $25.1 \%$, 从低龄级到高龄级的个体数逐步递减。

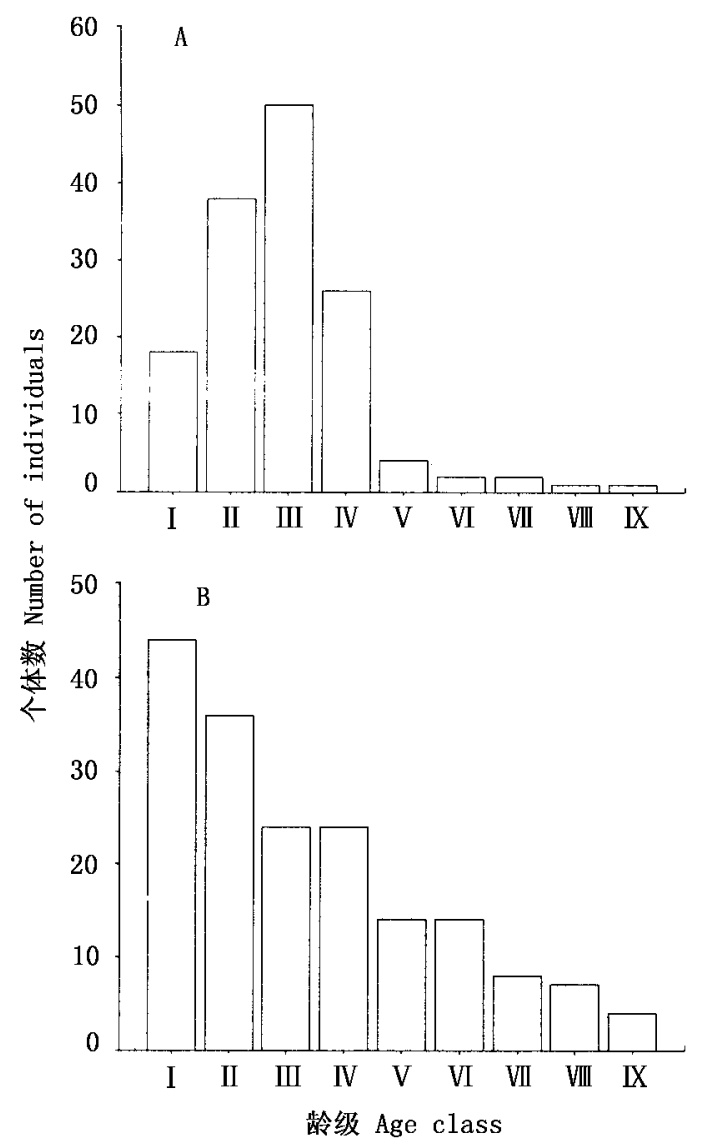

图 2 毒药树种群的年龄结构

Fig. 2 The age structures of two populations of Sladenia celastrifolia

A : 重度干扰种群 Heavily disturbed population B : 无干扰种群 Unaffected population

\section{2 毒药树种群静态生命表}

表 2 是两个种群的静态生命表,它反映了毒药 树种群生死规律的一些基本属性。从表 2 中我们可 
表 2 毒药树种群标准生命表

Table 2 The time-specific life table of two populations of Sladenia celastrifolia

\begin{tabular}{|c|c|c|c|c|c|c|c|c|c|}
\hline $\begin{array}{c}\text { 种群 } \\
\text { Population }\end{array}$ & $\begin{array}{c}\text { 龄级 } \\
\text { Age class }\end{array}$ & $\begin{array}{c}\text { 标准化存活数 } \\
l_{x} \\
\end{array}$ & $\begin{array}{c}\text { 死亡数 } \\
d_{x}\end{array}$ & $\begin{array}{c}\text { 死亡率 } \\
q_{x}\end{array}$ & $\begin{array}{c}\text { 期望寿命 } \\
e_{x}\end{array}$ & $\begin{array}{c}\text { 总寿命 } \\
T_{x}\end{array}$ & $\begin{array}{c}\text { 存活数 } \\
a_{x}\end{array}$ & $\ln l_{x}$ & $\begin{array}{c}\text { 消失率 } \\
K_{x}\end{array}$ \\
\hline \multirow[t]{9}{*}{ Population A } & I & 360 & -400 & -1111 & 7.39 & 2660 & 18 & 5.88 & -0.75 \\
\hline & II & 760 & -240 & -315 & 2.76 & 2100 & 38 & 6.63 & -0.27 \\
\hline & III & 1000 & 480 & 480 & 1.22 & 1220 & 50 & 6.90 & 0.65 \\
\hline & IV & 520 & 440 & 846 & 0.88 & 460 & 26 & 6.25 & 1.87 \\
\hline & V & 80 & 40 & 500 & 2.00 & 160 & 4 & 4.38 & 0.70 \\
\hline & VI & 40 & 0 & 0 & 2.50 & 100 & 2 & 3.68 & 0 \\
\hline & VII & 40 & 20 & 500 & 1.50 & 60 & 2 & 3.68 & 0.69 \\
\hline & VIII & 20 & 0 & 0 & 1.50 & 30 & 1 & 2.99 & 0 \\
\hline & IX & 20 & 0 & 0 & 0.50 & 10 & 1 & 2.99 & 0 \\
\hline \multirow[t]{9}{*}{ Population B } & I & 1000 & 182 & 182.0 & 3.48 & 3478.0 & 44 & 6.90 & 0.20 \\
\hline & II & 818 & 272 & 332.5 & 3.14 & 2569.0 & 36 & 6.70 & 0.40 \\
\hline & III & 546 & 0 & 0 & 3.45 & 1887.0 & 24 & 6.30 & 0 \\
\hline & IV & 546 & 228 & 417.5 & 2.45 & 1341.0 & 24 & 6.30 & 0.54 \\
\hline & V & 318 & 0 & 0 & 2.85 & 909.0 & 14 & 5.76 & 0 \\
\hline & VI & 318 & 136 & 427.6 & 1.85 & 591.0 & 14 & 5.76 & 0.56 \\
\hline & VII & 182 & 23 & 126.3 & 1.87 & 341.0 & 8 & 5.20 & 0.14 \\
\hline & VIII & 159 & 68 & 427.6 & 1.07 & 170.5 & 7 & 5.06 & 0.55 \\
\hline & IX & 91 & 0 & 0 & 0.50 & 45.5 & 4 & 4.51 & 0 \\
\hline
\end{tabular}

$l_{x}$ : Standard number of survivors at the age $x \quad d_{x}$ : Number of death during the interval age $(x, x+1) \quad q_{x}$ : Proportion of death during the interval age $(x, x+1) \quad e_{x}$ : Observed expectation of life at age $x \quad T_{x}$ : Life remaining to those at age $x \quad a_{x}$ : Actual number of alive individual at beginning of age $x \quad K_{x}$ : Killing power

以看出，受干扰严重的种群从 II 龄级到 VIII 龄级的死 亡率波动比较明显,而受干扰很少的种群的死亡率 相对比较稳定。

\section{3 毒药树种群存活曲线}

从图 3 我们可以看出, 种群 $\mathrm{A}$ 和种群 $\mathrm{B}$ 的存活 曲线存在很大的差异, 最大的不同点就是种群 $\mathrm{A}$ 的

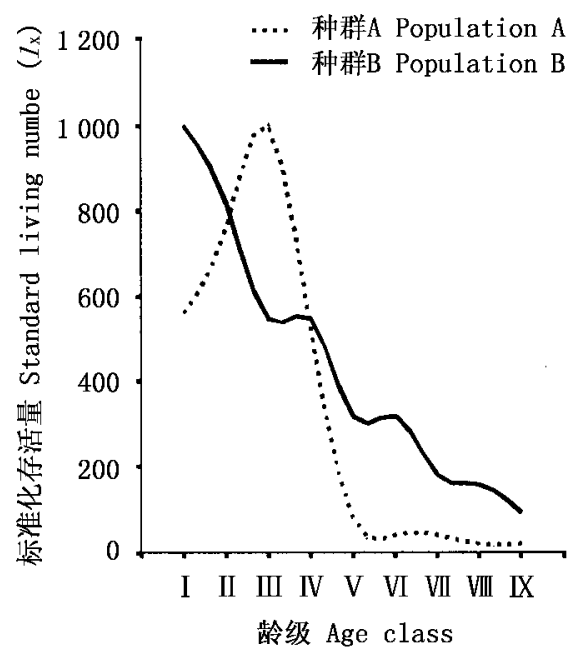

图 3 毒药树种群存活曲线

Fig.3 The survival curves of Sladenia celastrifolia
幼龄个体的死亡率为负值, 种群 $\mathrm{B}$ 各龄级个体的死 亡率的变化幅度不是很大。它们的一个共同的特征 就是低龄级个体的死亡率很高, 种群数量在早期就 急剧减少, 老龄个体的死亡率相对较低。与理想的 存活曲线相比 种群 $\mathrm{B}$ 的存活曲线表现出一定的波 动, 这可能是局部生境阶段性变化和气候波动的反 映。

\section{3 讨 论}

\section{1 人为干扰对种群数量特征的影响}

本文所研究的两个种群在年龄结构、生命表这 两方面都存在很大的不同，年龄结构及生命表是判 断植物种群发展趋势的重要指标 (Stewart,1986; Johnson \& Fryer , 1989)。受干扰严重的种群 A 的年 龄结构表现出很强的衰退趋势, 主要由以下几方面 的原因:在对森林公园进行建设的过程中，对毒药树 的砍伐和对幼苗的破坏就直接影响到了该种群的年 龄结构; 另外, 道路和景点的修建以及对毒药树的砍 伐破坏了毒药树所在的生态系统, 毒药树的生理及 生殖活动所依赖的群落内部环境就遭到了很大的改 变, 最后, 牲畜的践踏也对从根部萌生的幼苗造成了 很大的破坏。与之相对应, 种群 $\mathrm{B}$ 处在远离村寨的 
峡谷地带, 人类的破坏很少, 毒药树所依赖的生境保 持着相对比较原始的状态, 因此, 种群呈现出稳定的 特征。

通过比较两个种群的生命表可以看出, 种群 A 的 I 龄级和 II 龄级的死亡率为负, 说明幼苗库的量 不足 幼苗的补充对种群的发展起着决定性的作用， 因此，该种群持续发展受到很大的限制 整个种群的 维持依赖中龄个体 ( IV 龄级)，另外，VI龄级个体的高 死亡率与个体自身的生理衰老和人为的砍伐有很大 的关系。种群期望寿命 $\left(e_{x}\right)$ 反映了个体的平均生存 能力 种群 $\mathrm{A}$ 的期望寿命随着年龄的增长总体上呈 现不断降低的趋势, 说明种群的生存能力在逐级下 降。种群 $\mathrm{B}$ 受人为干扰的程度很小, I 龄级的 $e_{x}$ 最 高, 说明幼龄个体的生存能力处于最佳状态 种群的 继续发展具有稳定的后续资源，但高龄级个体的生 存能力相对较差。

受人为干扰严重的种群 $\mathrm{A}$ 的各项数量特征与 濒危植物鹅掌楸 (Liriodendron chinense) 和银杉 (Cathaya argyrophylla) 等(贺善安和郝日明, 1999 谢 宗强等 ,1999)具有相同的特点。通过对这两个种群 的比较可以看出，人类对其所依赖的生境的破坏是 导致毒药树种群走向衰退的主要原因。我们根据标 本记录, 通过一年多的实际调查发现, 毒药树的分布 点的个数也在逐渐减少。另外现存的种群所含个体 数都很少, 在所调查的 14 个种群中, 含个体数在 100 株以上的种群仅有 2 个 (即本研究中的 $A 、 B$ 种群)， 含个体数在 50 株以上 100 株以下的种群有 3 个。 物种分布区范围的收缩是该物种走向衰退的一个明 显标志(蒋志刚等, 1997)。从上述几个方面来看, 毒 药树是一个正在逐渐走向衰亡的物种。

\section{2 毒药树的保护}

虽然毒药树目前还未被列为濒危物种, 但国家 自然科学基金 八五” 重大项目 中国主要濒危植物 保护生物学研究” 所研究的 10 种典型濒危植物给予 了我们一些有益的启示。虽然这 10 种濒危植物所 处的地理环境各异, 濒危机理和濒危过程也不尽相 同，但通过对濒危机理和生态学过程进行分析，仍然 可以发现它们的许多共同性 (张文辉等,2002)。通 过对毒药树的研究发现, 受人为干扰严重的种群 A 的各项特征均与这 10 种典型濒危植物的相关特征 极为相似, 表现出衰退种群的特征, 而受人为干扰较 少的种群 B 却还相对比较稳定。按照世界自然保 护联盟 (IUCN) 的标准 (IUCN ,1994) 和有关濒危级别 的探讨(蒋志刚和枆恩源, 2003) , 我们认为, 毒药树
也应该被纳入受保护物种的范围。就地保护和迁地 保护是对物种保护的两种形式(蒋志刚等, 1997) , 迁 地保护是将物种移至人工环境或异地实施保护; 就 地保护就是在原来生境中对物种进行保护，也就是 对物种所依赖的生境和生态系统进行保护, 这也是 对物种进行保护的根本途径。但随着野生生物的生 存空间日益缩小, 迁地保护和就地保护有效的结合 将是对物种进行保护的一个必然趋势(蒋志刚等, 1997)。就毒药树的保护而言，我们认为应该采取就 地保护和迁地保护相结合的措施。首先是对现存的 野生毒药树种群进行就地保护, 将其纳入天然林保 护工程的范围 建立毒药树保护点, 进行有效的怃育 和管理 ;其次是根据毒药树的生态习性，选择适宜的 地点，在植物园内进行种植，建立人工种群。将这两 个方面结合，才能达到对毒药树的种质资源有效保 护的目的。

\section{参 考 文 献}

Bruna EM (2003) . Are plant populations in fragmented habitats recruitment limited? Tests with an Amazonian herb. Ecology, 84, $932-947$.

Guo HJ (郭辉军) (1998). Biodiversity in Yunnan (云南的生物 多样性). Yunnan Science and Technology Press, Kunming. (in Chinese)

He SA (贺善安), Hao RM (郝日明) (1999). Study on the nature population dynamics and the endangered habitat of Liriodendron chinense in China. Acta Phytoecologica Sinica (植物生态 学报),23,87-95. (in Chinese with English abstract)

IUCN ( 1994 ). IUCN Red List Categories. Switzerland, IUCN Gland.

Jiang H (江洪) (1992). The Population Ecology of Picea asperat (云杉种群生态学). China Forestry Publishing House, Beijing, 6-24. (in Chinese)

Jiang ZG (蒋志刚), Fan EY (樊恩源) (2003). Exploring the endangered species criteria: rethinking the IUCN Red List Criteria. Biodiversity Science (生物多样性), 11, 383 - 392.（in Chinese with English abstract)

Jiang ZG (蒋志刚), Ma KP (马克平), Han XG (韩兴国) (1997). Conservation Biology (保护生物学). Zhejiang Science and Technology Press, Hangzhou, 120 - 131. (in Chinese with English abstract)

Johnson EA, Fryer GI (1989) . Population dynamics in Longdgepole Pine-Engelmann spruce forest. Ecology, 70, 1335 - 1345 .

Li L (李璐) (2001). Chromosome number of Sladenia celastrifolia. Acta Botanica Yunnanica (云南植物研究) ,23, 223-224. (in Chinese with English abstract)

Scariot A (1999) . Forest fragmentation effects on palm diversity in central Amazonia. Journal of Ecology, 87, 66-76. 
Stewart GH (1986) . Population dynamics of a montane conifer forest, western Cascade Range, Oregon. USA. Ecology, 67, 534 -544 .

Wang XK, Feng ZW, Ouyang ZY (2001). The impact of human disturbance on vegetative carbon storage in forest ecosystems in China. Forest Ecology and Management, 148, 117 - 123.

Wu CZ (吴承祯), Hong W(洪伟) (2002). A proposed multidimensional time series model of individual age and diameter in Tsuga longibracteata. Acta Phytoecologica Sinica (植物生态学 报), 26, 403-407. (in Chinese with English abstract)
Xie ZQ (谢宗强), Chen WL (陈伟烈)，Lu P(路鹏)，Hu D (胡东) (1999). Population statistics and age structure of endangered plant Cathaya argyrophylla. Acta Ecologica Sinica (生态 学报), 19,523-528. (in Chinese with English abstract) Zhang WH (张文辉)，Zu YG (祖元刚), Liu GB (刘国彬) (2002). Population ecological charactertics and analysis on endangered cause of ten endangered plant species. Acta Ecologica Sinica (生态学报), 22,1512-1520. (in Chinese with English abstract) 